\title{
Osteonecrosis of the jaws in dogs in previously irradiated fields: 13 cases (1989-2014)
}

\section{Ana Nemec ${ }^{1,2 *}$, Boaz Arzi ${ }^{2}$, Katherine Hansen ${ }^{2}$, Brian G. Murphy ${ }^{3}$, Milinda J. Lommer ${ }^{2,4}$, Santiago Peralta ${ }^{5}$ and Frank J. M. Verstraete ${ }^{2}$}

${ }^{1}$ Clinic for Surgery and Small Animals, Veterinary Faculty, University of Ljubljana, Ljubljana, Slovenia

2 Department of Surgical and Radiological Sciences, School of Veterinary Medicine, University of California at Davis, Davis, CA, USA

${ }^{3}$ Department of Pathology, Microbiology and Immunology, School of Veterinary Medicine, University of California at Davis, Davis, CA, USA

${ }^{4}$ Aggie Animal Dental Center, Mill Valley, CA, USA

${ }^{5}$ Department of Clinical Sciences, College of Veterinary Medicine, Cornell University, Ithaca, NY, USA

\section{Edited by:}

Christopher R. Byron

Virginia-Maryland College of

Veterinary Medicine, USA

Reviewed by:

Kevin S. Stepaniuk, Columbia River Veterinary Specialists, USA

Neil Christensen, University of

Wisconsin - Madison, USA

*Correspondence:

Ana Nemec, Clinic for Surgery and

Small Animals, Veterinary Faculty,

University of Ljubljana, Cesta v

Mestni Log 47, Ljubljana 1000,

Slovenia

e-mail: ana.nemec@vf.uni-lj.si
The aim of this report was to characterize osteonecrosis of the jaws (ONJ) in previously irradiated fields in dogs that underwent radiotherapy (RT) for oral tumors. Osteoradionecrosis of the jaw (ORNJ) was further defined as osteonecrosis in a previously irradiated field in the absence of a tumor. Thirteen dogs clinically diagnosed with $15 \mathrm{ONJ}$ lesions were included in this retrospective case series. Medical records were reviewed for: breed, sex, weight, and age of the patient, tumor type, location in the oral cavity and size, location of the ONJ, time from RT to ONJ onset, known duration of the ONJ, and tumor presence. Where available, histological assessment of tissues obtained from the primary tumor, and tissues obtained from the ONJ lesion, was performed, and computed tomographic (CT) images and dental radiographs were reviewed. RT and other treatment details were also reviewed. Twelve dogs developed ONJ in the area of the previously irradiated tumor or the jaw closest to the irradiated mucosal tumor. Recurrence of neoplasia was evident at the time of ONJ diagnosis in five dogs. Time from RT start to ONJ onset varied from 2 to 44 months. In three cases, ORNJ developed after dental extractions in the irradiated field. Dental radiographs mostly revealed a moth-eaten pattern of bone loss, CT mostly revealed osteolysis, and histopathology was consistent with osteonecrosis. To conclude, development of ONJ/ORNJ following $\mathrm{RT}$ is a rare, but potentially fatal complication. Patients undergoing RT may benefit from a comprehensive oral and dental examination and treatment prior to RT.

Keywords: dog, jaw osteonecrosis, oral tumors, osteoradionecrosis, radiotherapy

\section{INTRODUCTION}

Radiotherapy (RT) is an important treatment modality for oral tumors in humans (1) and dogs (2-4); however, the irradiated patient is susceptible to developing early and long-term complications due to radiation (1-5). Osteoradionecrosis (ORN) of the jaw (ORNJ) is a devastating long-term complication of RT in humans that leads to reduced quality of life due to pain, dysphagia, facial deformation, and may even result in death $(1,6-8)$. Several theories about ORNJ pathogenesis have been suggested, with the latest considering bone damage being caused by radiation-induced fibrosis due to acute inflammation, production of free radicals, and chronic activation of fibroblasts (1). The reported incidence of ORNJ in humans ranges from 2 to $8 \%$ with a reported decline in recent years $(1,6-8)$. Late complications of RT in dogs are also rare (2), although the reported frequency of ORN is variable. In appendicular sites, ORN was reported in $4 \%$ of cases treated with orthovoltage RT in one study (9). However, Théon et al. (3, 4) reported that $6.4-7.6 \%$ of cases treated with megavoltage RT for canine oral tumors experienced ORNJ. Although orthovoltage is rarely used in current veterinary RT, Thrall (10) reported that $5 \%$ of dogs developed ORNJ after orthovoltage RT for canine acanthomatous ameloblastoma (CAA).
The diagnosis of ORNJ in humans is based primarily on clinical signs; usually ulceration of the mucosa with exposure of necrotic bone is noted. However, additional definitions and classifications have been employed, mainly depending on the persistence or recurrence of a primary tumor, radiologic signs, duration of bone exposure, and extent of the disease $(1,6,11-13)$. Several factors predisposing a patient for ORNJ development have also been described in human medicine $(1,6,7,14)$, but reports are rare in veterinary medicine.

The aim of this retrospective study was to characterize osteonecrosis of the jaws (ONJ) in previously irradiated fields in a series of dogs that underwent RT for oral tumors as this entity is poorly defined in the veterinary literature. ORN of the jaw (ORNJ) was specifically defined for the purpose of this study as osteonecrosis in a previously irradiated field in the absence of persistent or recurrent tumor.

\section{MATERIALS AND METHODS SELECTION CRITERIA}

Medical records from the small animal clinic of the William R. Pritchard Veterinary Medical Teaching Hospital of the University of California Davis, were searched for dogs that had diagnostic 
and/or treatment procedures for clinically diagnosed ONJ based upon clinical signs of ulceration of the mucous membrane with exposure of necrotic bone, regardless of tumor presence or recurrence. Ten cases met the inclusion criteria and were included in this study. Two additional cases were evaluated at Aggie Animal Dental Center, Mill Valley, CA, USA, and one additional case at the Department of Clinical Sciences, College of Veterinary Medicine, Cornell University, resulting in a total of 13 cases included in this study.

This investigation did not seek to identify the incidence of ONJ/ORNJ lesions in previously irradiated fields. The cases series reported here represent patients with ONJ/ORNJ who were seen at three different veterinary dentistry and oral surgery referral hospitals, regardless of where in the USA the cases were treated with RT.

\section{MEDICAL RECORDS REVIEW}

Medical records were reviewed, and the following data were recorded: breed, sex, weight, age of the patient at the start of RT, tumor type, location in the oral cavity and size $(T)$ (15), location of the ONJ, time from RT to ONJ onset, and known duration of the ONJ. Time from RT to ONJ onset was determined as the time interval between the start of the (first) RT fraction and the onset of clinical signs typical of ONJ (ulceration of the mucosa with exposure of necrotic bone). Additionally, tumor presence or recurrence was recorded, and lesions where none was reported were considered "true" ORNJ (1). Known duration of the ONJ (i.e., duration of bone exposure) was determined as the interval between lesion identification (either by the client, referring veterinarian, or at the referral hospital) to the time of healing following surgical intervention, death, or last available follow-up. In cases, where the histology slides were available for review, histological assessment of the primary tumor before RT and of the tissues obtained from the site of the clinically determined ONJ, was performed. Additionally, computed tomographic (CT) images, if available at initial presentation (before RT) and at ONJ clinical diagnosis, were evaluated on a medical grade flat-screen monitor with commercially available software (eFilm Work station 3.4, eFilm Medical Inc., Toronto, ON, Canada). Dental radiographs were also reviewed. Similarly, RT details were also reviewed for information on radiation planning and delivery, and any additional treatments before and/or during the RT were also recorded.

\section{RESULTS \\ POPULATION}

Over a 25-year period (1989-2014), 13 dogs were clinically diagnosed with 15 ONJ lesions in previously irradiated fields (Table 1). Six dogs were mixed-breed, two were labrador retrievers, and one each of the following breeds: golden retriever, beagle, bloodhound, bichon frise, and brittany spaniel. There were five spayed females and eight castrated males. Dogs ranged in weight from 12.6 to $40 \mathrm{~kg}$ (median $25.5 \mathrm{~kg}$, mean $25.4 \mathrm{~kg}$ ). Dogs were $8-17$ years old (median 11 years, mean 10.8 years) at the start of RT.

\section{CHARACTERISTICS OF THE PRIMARY TUMOR}

The dogs were treated for each of CAA $(N=4)$, squamous cell carcinoma (SCC, $N=4$ ), and melanoma (MM, $N=4)$, and for an undifferentiated sarcoma $(N=1)$. Slides of the primary neoplastic lesions were available for review in only three cases, with all other tumors diagnosed by outside commercial laboratories. In the three cases with slides available, the neoplasia diagnosis was confirmed histologically by a board-certified pathologist (BGM). Location and size of the oral tumors are presented in Table 1.

Pre-RT imaging (dental radiographs, skull radiographs, and/or CT) were available for six of the dogs (all T3 cases and one T2 case) and revealed bony invasion in three of the T3 cases. Data on the size of the tumors were not available for two dogs.

For radiation treatments, patients were treated using a Cobalt60 (Co-60 unit) (El Dorado 8, AECL, Montreal, QC, Canada), $4 \mathrm{MV}$ linear accelerator (Clinac 4, Varian, Palo Alto, CA, USA), or Clinac 2100 (Varian, Palo Alto, CA, USA). The source used for treatment of the Case 9 is unknown. Patients were treated with $32-54$ Gy in 3-8 Gy fractions depending on the clinical radiation plan. The total number of fractions delivered ranged from 3 to 17 fractions. A total of four patients were treated with a palliative plan $(4 \times 8$ Gy on a once- or twice-weekly basis $)$ for gross CAA, MM, and SCC. A total of six patients were treated with a more coarsely fractionated definitive protocol $(7 \times 6 \mathrm{~Gy}$ once weekly or $12 \times 4$ Gy Monday-Wednesday-Friday) for CAA and MM. Three cases were treated with more typical fractionated definitive protocols $(16 \times 3 \mathrm{~Gy}$ or $18 \times 3 \mathrm{~Gy})$ for SCC and undifferentiated sarcoma. One patient received both a coarsely fractionated definitive protocol $(12 \times 4 \mathrm{~Gy})$, followed by a palliative protocol 7 months later (Tables $2-4$ ). Nine patients were treated with a hand-calculated plan, while four patients received a computer-based plan using 3D-conformal planning. Eleven patients had gross disease visible at the time of RT treatment, and three patients had gross disease noted in the record at the end of RT (Table 1). Both of the dogs without gross disease received definitive-intent radiation protocols, while those patients with gross disease received either palliative or definitive-intent protocols.

One patient had a treatment interruption of 13 days between fractions seven and eight, and the eighth fraction was given as a dose of $6 \mathrm{~Gy}$; the reason is unknown. One patient received only five out of six planned doses. One patient received its first dose as a single field, after which the treatment was changed to a parallel-opposed two-field technique for subsequent fractions; the last treatment was not delivered due to poor patient condition. One patient received 3 Gy on its first definitive treatment day, but thereafter received $4 \mathrm{~Gy}$ fractions. The American College of Veterinary Radiology-Radiation Oncology recommended reporting data for radiation studies can be found in Tables 2-4 (16).

\section{OTHER TREATMENTS}

Prior to RT, three dogs had their oral masses surgically reduced, including bone excision in one of these dogs. The other 10 dogs received no surgical intervention (other than biopsy) prior to RT. The stage of periodontal disease was not established in the majority of cases before RT, but five dogs received full periodontal treatment up to 3 months prior to initiation of RT.

Eleven dogs were treated with an antibiotic during or immediately after RT, but the timing and antibiotic selected varied. 
Table 1 | General characteristics of the primary tumor and osteonecrosis lesion.

\begin{tabular}{|c|c|c|c|c|c|c|c|c|c|c|c|c|}
\hline Case & Tumor location & $\begin{array}{l}\text { Tumor } \\
\text { type }\end{array}$ & T stage & $\begin{array}{l}\text { Gross } \\
\text { tumor } \\
\text { disease } \\
\text { at RT } \\
\text { start }\end{array}$ & $\begin{array}{l}\text { Gross } \\
\text { tumor } \\
\text { disease } \\
\text { at RT } \\
\text { end }\end{array}$ & $\begin{array}{l}\text { Lesion } \\
\text { location }\end{array}$ & $\begin{array}{l}\text { Imaging } \\
\text { of the } \\
\text { lesion } \\
\text { CT/dental } \\
\text { radiographs }\end{array}$ & Histopathology & $\begin{array}{l}\text { Time to lesion } \\
\text { onset }\end{array}$ & $\begin{array}{l}\text { Known } \\
\text { lesion } \\
\text { duration }\end{array}$ & $\begin{array}{l}\text { Tumor } \\
\text { recurrence } \\
\text { at lesion } \\
\text { onset }\end{array}$ & Outcome \\
\hline 1 & $\begin{array}{l}\text { L caudal maxilla } \\
\text { (P3-P4) }\end{array}$ & SCC & T1 & No & No & $\begin{array}{l}\text { L caudal } \\
\text { maxilla }\end{array}$ & No/yes & No & $\begin{array}{l}15.5 \text { months, } \\
\text { post-extractions }\end{array}$ & 1 month & No & Lost to follow-up \\
\hline 2 & R buccal mucosa & MM & N/A & Yes & No & $\begin{array}{l}\text { R caudal } \\
\text { maxilla }\end{array}$ & No/no & $\begin{array}{l}\text { Yes - soft tissues } \\
\text { only }\end{array}$ & $\begin{array}{l}3.5 \text { months, } \\
\text { post-extractions }\end{array}$ & 3 months & No & Lost to follow-up \\
\hline 3 & $\begin{array}{l}\text { L caudal mandible } \\
\text { (M1, lingual) }\end{array}$ & CAA & $\mathrm{T} 1$ & Yes & No & $\begin{array}{l}R \text { caudal } \\
\text { mandible }\end{array}$ & Yes/yes & Yes & 17 months & 3 months & No & $\begin{array}{l}\text { Remission after } \\
\text { mandibular rim excision }\end{array}$ \\
\hline 4 & $\mathrm{~L}$ caudal maxilla & CAA & T3b & Yes & No & $\begin{array}{l}\text { L caudal } \\
\text { maxilla }\end{array}$ & $\begin{array}{l}\text { Yes/yes - at } \\
\text { the time of } \\
\text { ONJ revision }\end{array}$ & $\begin{array}{l}\text { Yes - soft tissues } \\
\text { only }\end{array}$ & 4 months & 16 months & No & $\begin{array}{l}\text { Stable disease after } \\
\text { several débridements }\end{array}$ \\
\hline 5 & $\begin{array}{l}\text { Hard-soft palate } \\
\text { junction }\end{array}$ & MM & $\mathrm{T} 2$ & No & No & $\begin{array}{l}\text { L caudal } \\
\text { maxilla; later } \\
\mathrm{R} \text { caudal } \\
\text { maxilla }\end{array}$ & $\begin{array}{l}\text { Yes/yes; } \\
\text { yes/yes }\end{array}$ & $\begin{array}{l}\text { Yes; yes - soft } \\
\text { tissues only }\end{array}$ & $\begin{array}{l}\text { L side: } 26 \text { months } \\
\text { ( } 9 \text { months } \\
\text { post-extractions); } \\
\text { R side: } 44 \text { months }\end{array}$ & $\begin{array}{l}\text { L side: } \\
2 \text { months; } \\
\text { R side: } \\
1 \text { month }\end{array}$ & No & $\begin{array}{l}\text { L side: remission after } 2 \\
\text { débridements; } R \text { side: } \\
\text { remission after } \\
\text { débridement }\end{array}$ \\
\hline 6 & $\begin{array}{l}\mathrm{L} \text { caudal oral } \\
\text { cavity/oro-pharynx }\end{array}$ & MM & T3a & Yes & No & $\begin{array}{l}\mathrm{L} \text { caudal } \\
\text { mandible }\end{array}$ & No/yes & $\begin{array}{l}\text { Yes - soft tissues } \\
\text { only }\end{array}$ & 6 months & 1.5 months & No & $\begin{array}{l}\text { Progressive lesion, } \\
\text { euthanasia }\end{array}$ \\
\hline 7 & Sublingual & MM & $\mathrm{T} 1$ & Yes & No & $\begin{array}{l}L \text { and } R \\
\text { caudal } \\
\text { mandibles }\end{array}$ & No/yes & $\begin{array}{l}\text { Yes - not available } \\
\text { for review }\end{array}$ & 13 months & $<1$ month & No & Lost to follow-up \\
\hline 8 & R caudal maxilla & SCC & $\mathrm{T} 1$ & Yes & No & $\begin{array}{l}\mathrm{R} \text { caudal } \\
\text { maxilla }\end{array}$ & No/yes & $\begin{array}{l}\text { Yes - not available } \\
\text { for review }\end{array}$ & 3 months & 2 months & No & $\begin{array}{l}\text { Managed medically for } \\
6 \text { months prior to } \\
\text { euthanasia }\end{array}$ \\
\hline 9 & R caudal mandible & SCC & N/A & Yes & No & $\begin{array}{l}\text { R caudal } \\
\text { mandible }\end{array}$ & Yes/yes & No & 2 months & 1 month & Yes & Euthanasia suggested \\
\hline 10 & $\begin{array}{l}\text { L caudal maxilla } \\
\text { (P3-M2) }\end{array}$ & CAA & T3b & Yes & Yes & $\begin{array}{l}\text { L caudal } \\
\text { maxilla }\end{array}$ & No/no & No & 13 months & 6 months & Yes & $\begin{array}{l}\text { Slowly progressive lesion, } \\
\text { euthanasia suggested }\end{array}$ \\
\hline 11 & $\begin{array}{l}\text { R caudal mandible } \\
\text { (M1-M3, crossing } \\
\text { midline) }\end{array}$ & SCC & T3b & Yes & No & $\begin{array}{l}\mathrm{R} \text { caudal } \\
\text { mandible }\end{array}$ & No/no & No & 6.5 months & 1 month & Yes & Lost to follow-up \\
\hline 12 & $\begin{array}{l}\text { R caudal mandible } \\
\text { (P3-M1) }\end{array}$ & CAA & $\mathrm{T} 2$ & Yes & Yes & $\begin{array}{l}\mathrm{R} \text { caudal } \\
\text { mandible }\end{array}$ & Yes/yes & Yes & 11 months & 3 months & Yes (SCC) & $\begin{array}{l}\text { Remission after } 2 \mathrm{nd} \\
\text { resection (R rostral } \\
\text { mandibulectomy) }\end{array}$ \\
\hline 13 & L caudal mandible & $\begin{array}{l}\text { Undiffer- } \\
\text { entiated } \\
\text { sarcoma }\end{array}$ & T3а & Yes & Yes & $\begin{array}{l}\text { L caudal } \\
\text { mandible }\end{array}$ & Yes/yes & No & 8.5 months & 1 week & Yes & Euthanasia suggested \\
\hline
\end{tabular}

L, left; R, right; P, premolar tooth; M, molar tooth; SCC, squamous cell carcinoma; MM, melanoma; CAA, canine acanthomatous ameloblastoma; T1, tumor smaller than 20 mm in maximum diameter; T2, tumor 20-40 mm in maximum diameter; $T 3$, tumor larger than $40 \mathrm{~mm}$ maximum diameter (a, without bone involvement; $b$, with bone involvement). 
Table 2 | Reporting of radiation prescription and target doses as recommended by the American College of Veterinary Radiology-Radiation Oncology (ACVR-RO).

\begin{tabular}{|c|c|c|c|c|c|c|c|}
\hline $\begin{array}{l}\text { Patient } \\
\text { number }\end{array}$ & RT intent & $\begin{array}{l}\text { Dose per } \\
\text { fraction (Gy) }\end{array}$ & $\begin{array}{l}\text { Intended total } \\
\text { dose (Gy) }\end{array}$ & $\begin{array}{l}\text { Prescription } \\
\text { point of dose }\end{array}$ & $\begin{array}{l}\text { Target dose and dose } \\
\text { variation within plan }\end{array}$ & $\begin{array}{l}\text { Number of } \\
\text { fractions }\end{array}$ & $\begin{array}{l}\text { Time } \\
\text { schedule }\end{array}$ \\
\hline 1 & Definitive & 3 & 48 & $1 \mathrm{~cm}$ & $\begin{array}{l}\text { DMAX }=3.11 \mathrm{~Gy} \text {, exit } \\
\text { dose }=2.59 \mathrm{~Gy} \text {; treat to } \\
100 \% \text { isodose line }\end{array}$ & 16 & Daily \\
\hline 2 & $\begin{array}{l}\text { Coarsely fractionated, } \\
\text { definitive intent }\end{array}$ & 6 & 42 & $3 \mathrm{~cm}$ & Skin dose $=6.9 \mathrm{~Gy}$ & 7 & $\begin{array}{l}\text { Twice } \\
\text { weekly }\end{array}$ \\
\hline 4 & $\begin{array}{l}\text { Coarsely fractionated, } \\
\text { definitive intent }\end{array}$ & 4 & 48 & $5 \mathrm{~cm}$ & $\begin{array}{l}\mathrm{DMAX}=4.86 \mathrm{~Gy}, \text { Skin } \\
\text { dose }=3.99 \mathrm{~Gy}\end{array}$ & 12 & MWF \\
\hline 5 & $\begin{array}{l}\text { Coarsely fractionated, } \\
\text { definitive intent }\end{array}$ & 4 & 48 & $\begin{array}{l}\text { PTV; normalize } \\
\text { to } 90 \%\end{array}$ & $\begin{array}{l}\text { MIN for PTV: } 78.3 \% \text {; MAX } \\
\text { for PTV: } 110.4 \% \text {; mean for } \\
\text { PTV: } 95.7 \% \text {; }\end{array}$ & 12 & MWF \\
\hline 8 & Palliative & 8 & 32 & $5 \mathrm{~cm}$ & Treat to $100 \%$ isodose line & 4 & $\begin{array}{l}\text { Twice } \\
\text { weekly }\end{array}$ \\
\hline 9 & Definitive & 3 & 54 & $\begin{array}{l}\text { PTV; } 100 \% \\
\text { dose }\end{array}$ & $\begin{array}{l}\mathrm{DMAX}=3.23 \mathrm{~Gy}, \text { isocenter } \\
\text { dose }=3 \mathrm{~Gy}\end{array}$ & 18 & Daily \\
\hline 10 & $\begin{array}{l}\text { Coarsely fractionated, } \\
\text { definitive } \\
\text { intent + Palliative } \\
7 \text { months after } \\
\text { definitive }\end{array}$ & $\begin{array}{l}4 \text { for definitive; } \\
\text { unknown for } \\
\text { follow-up } \\
\text { palliative } \\
\text { protocol }\end{array}$ & $\begin{array}{l}48 \text { for definitive; } \\
\text { unknown for } \\
\text { follow-up } \\
\text { palliative } \\
\text { protocol }\end{array}$ & $\begin{array}{l}\text { Record not } \\
\text { available }\end{array}$ & Record not available & $\begin{array}{l}12 \text { for } \\
\text { definitive; } 5 \\
\text { for palliative }\end{array}$ & $\begin{array}{l}\text { MWF for } \\
\text { definitive; } \\
\text { twice } \\
\text { weekly for } \\
\text { palliative }\end{array}$ \\
\hline 13 & Definitive & 3 & 48 & $\begin{array}{l}\text { PTV, normalize } \\
\text { to } 88 \%\end{array}$ & $\begin{array}{l}\text { MIN for PTV: } 29 \% \text {; MAX for } \\
\text { PTV: } 117.2 \% \text {; mean for } \\
\text { PTV: } 109 \%\end{array}$ & 16 & Daily \\
\hline
\end{tabular}

MWF, Monday, Wednesday, Friday treatment days; GTV, gross tumor volume; CTV, clinical target volume; PTV, planning target volume; DMAX, depth of dose maximum; MIN, minimum dose; MAX, maximum dose.

Only one dog received regular chlorhexidine gluconate oral rinses, starting in the middle of the RT when mucositis developed. In two dogs, a tea rinse was started at the time of mucositis development.

Three dogs received intratumoral chemotherapy (substance unknown), and five additional dogs received systemic chemotherapy, or a small molecule inhibitor (carboplatin, iniparib, or doxorubicin), or melanoma vaccine (Oncept, Merial) during RT or shortly after the RT was completed.

Seven dogs were treated with prednisone, with the treatment initiated during RT in six dogs and at the beginning of RT in one dog. The use of analgesics varied, but typically included an opioid and a non-steroidal anti-inflammatory drug in cases that were not treated with a steroid.

\section{CHARACTERISTICS OF THE ONJ LESIONS}

Clinically, ONJ lesions were characterized as bone and soft tissue necrosis of variable extent (1) (Figure 1), regardless of evidence of tumor recurrence, and such lesions were detected in 13 cases. Recurrence of neoplasia (Figure 2) was evident on clinical and/or histopathological examination at the time of ONJ diagnosis in five dogs. All other cases were considered to be consistent with a diagnosis of ORNJ (Table 1). 
Table 3 | Reporting of radiation planning and target volumes as recommended by the ACVR-RO.

\begin{tabular}{|c|c|c|c|c|c|c|}
\hline $\begin{array}{l}\text { Patient } \\
\text { number }\end{array}$ & $\begin{array}{l}\text { Plan } \\
\text { type }\end{array}$ & $\begin{array}{l}\text { TPS } \\
\text { used }\end{array}$ & $\begin{array}{l}\text { Heterogeneity } \\
\text { correction }\end{array}$ & GTV & CTV & PTV \\
\hline 1 & Manual & N/A & N/A & No gross tumor present & Not defined & Lmax P3-P4 + $3 \mathrm{~cm}$ \\
\hline 2 & Manual & N/A & N/A & Pinpoint lesion & Not defined & Not defined \\
\hline 3 & Manual & N/A & N/A & $1 \mathrm{~cm}$ mass Lmand M1 & Not defined & Not defined \\
\hline 4 & Manual & N/A & N/A & $\begin{array}{l}\text { L caudal maxilla, eroded } \\
L \text { zygomatic bone, and } \\
\text { into } L \text { nasal cavity }\end{array}$ & Not defined & Not defined \\
\hline 5 & Computer & Eclipse & Yes & Not defined & $\begin{array}{l}\text { Hard-soft palate junction, just } L \text { to the } \\
\text { midline, going caudally to } 1 \mathrm{~cm} \text { proximal } \\
\text { to the hyoid process }\end{array}$ & $\begin{array}{l}\text { PTV }=\mathrm{CTV}+4 \mathrm{~mm} \\
\text { Volume } 9.52 \mathrm{~cm}^{3}\end{array}$ \\
\hline 6 & Manual & Eclipse & $\mathrm{N} / \mathrm{A}$ & Lmand M1-M3 & Not defined & Lmand M1-M3 + $3 \mathrm{~cm}$ \\
\hline 7 & Computer & Eclipse & Yes & Not defined & Not defined & volume $31.1 \mathrm{~cm}^{3}$ \\
\hline 8 & Manual & N/A & N/A & Not defined & Not defined & Not defined \\
\hline 9 & Manual & N/A & $\mathrm{N} / \mathrm{A}$ & Not defined & Not defined & Not defined \\
\hline 10 & Manual & N/A & $\mathrm{N} / \mathrm{A}$ & Record not available & Record not available & Record not available \\
\hline 11 & Computer & Eclipse & No & Not defined & $\begin{array}{l}\text { Rmand P4-ramus, with lateral and ventral } \\
\text { ST to midline. } 158.19 \mathrm{~cm}^{3}\end{array}$ & $\begin{array}{l}\mathrm{PTV}=\mathrm{CTV}+3 \mathrm{~mm} \\
\text { volume }=211.68 \mathrm{~cm}^{3}\end{array}$ \\
\hline 12 & Manual & $\mathrm{N} / \mathrm{A}$ & N/A & $3 \mathrm{~cm} \times 2 \mathrm{~cm} \times 1.8 \mathrm{~cm}$ & Not defined & $\mathrm{GTV}+3 \mathrm{~cm}$ \\
\hline 13 & Computer & Eclipse & Yes & Not defined & $\begin{array}{l}\text { Left caudal mandible, level of } \mathrm{P} 1-\mathrm{M} 3 \text {, } \\
\text { including ramus and tip of Max } \mathrm{M} 1-\mathrm{M} 2 \text {, } \\
\text { with surrounding lateral soft tissue and } \\
\text { ventral soft tissue to midline; } 66.96 \mathrm{~cm}^{3}\end{array}$ & $\begin{array}{l}\mathrm{PTV}=\mathrm{CTV}+1 \mathrm{~cm} \\
\text { volume } 192.33 \mathrm{~m}^{3}\end{array}$ \\
\hline
\end{tabular}

Co-60, Cobalt-60 radiotherapy unit; CTV, clinical target volume; PTV, planning target volume; TPS, treatment planning system.

As presented in Table 1, eight dogs developed lesions in the area of the previously irradiated tumor. Four dogs developed ORNJ lesion in the jaw adjacent to the irradiated tumor, and in one dog the ORNJ was found on the contralateral mandible. In one dog with a maxillary ORNJ lesion, an oronasal fistula (ONF) was noted at presentation, and in two additional dogs ONF developed after débridement. In two of these dogs, an orocutaneous fistula also developed.

Time from RT to ONJ onset varied from 2 to 44 months (median 9.8 months, mean 12.4 months), and for ORNJ specifically 3 to 44 months (median 13 months, mean 14.7 months) (Table 1). In one dog, ORNJ was noted 26 months after initiation of RT and 9 months after dental extractions were performed within the irradiation field; retained roots were later diagnosed. The same dog later developed ORNJ of the contralateral maxilla. Dental extractions were performed in the irradiated field in two other animals, with subsequent development of ORNJ.

Known duration of the ONJ varied from 1 week to 16 months (median 1.8 months, mean 2.9 months), and for ORNJ specifically from less than 1 to 16 months (median 2 months, mean 3.4 months) (Table 1 ).

Dental radiographs from the time of ONJ diagnosis were available for 10 dogs (11 ONJ/ORNJ lesions). With the exception of one dog with bony changes in the area of the clinical lesion limited to horizontal bone loss associated with periodontitis, dental radiographs consistently revealed a moth-eaten pattern of bone loss (17). In six dogs, this moth-eaten bone loss was combined with a geographic and/or permeative pattern of bone loss (17). In three dogs where the mandible was involved, increased bone density with generalized loss of definition of the mandibular canal was diagnosed, with a solid periosteal reaction (17) noted in one dog (Figure 3). On CT, findings associated with the ONJ/ORNJ lesion were described as extensive and incomplete or irregular osteolysis (Figure 4) in five of six dogs, with one additional case demonstrating diffuse thinning of the mandible with multifocal pitting osteolysis (Figure 5).

Histopathology was performed in eight dogs with clinically diagnosed ONJ/ORNJ lesions, but specimens were only available for review in six dogs, including a dog where two biopsies of a progressive ONJ lesion were obtained 2 months apart. Bone and soft tissues were available for evaluation in three dogs, and only soft tissue was available for the other lesions. Soft tissue changes in all but one dog included pleocellular inflammation with mucosal hyperplasia and/or dysplasia and diffuse subepithelial fibrosis with multifocal vasculopathy and thrombosis. In one dog, the changes were most consistent with suppurative inflammation associated with an extensive area of necrotic tissue, and likely vascular thrombosis. Changes in the bone were described as locally extensive osteonecrosis with bone resorption and osteoporosis (Figure 6). Two of these lesions demonstrated features of osteomyelitis or had an attached biofilm on the exposed bone surface. In one dog, recurrent CAA was confirmed at the time of ONJ clinical diagnosis and was surgically removed with tumorfree margins (segmental mandibulectomy). However, 2 months later ( 13 months after the start of RT), the ONJ lesion was progressed and a right rostral mandibulectomy was performed. The 
Table 4 | Reporting of treatment delivery and beam information as recommended by the ACVR-RO.

\begin{tabular}{|c|c|c|c|c|c|c|c|c|c|c|}
\hline $\begin{array}{l}\text { Patient } \\
\text { number }\end{array}$ & $\begin{array}{l}\text { Treatment } \\
\text { interruptions }\end{array}$ & $\begin{array}{l}\text { Deviation } \\
\text { from } \\
\text { protocol }\end{array}$ & $\begin{array}{l}\text { Beam } \\
\text { quality } \\
\text { and } \\
\text { energy }\end{array}$ & Equipment & $\begin{array}{l}\text { SSD/ } \\
\text { SAD }\end{array}$ & Technique & $\begin{array}{l}\text { Beam } \\
\text { weighting }\end{array}$ & $\begin{array}{l}\text { Field } \\
\text { size }(\mathrm{cm})\end{array}$ & $\begin{array}{l}\text { Beam } \\
\text { modification } \\
\text { and bolus }\end{array}$ & $\begin{array}{l}\text { Skin } \\
\text { bolus }\end{array}$ \\
\hline 1 & No & No & $6 \mathrm{MV}$ & Clinac 2100 & SSD & Single field & N/A & $4.8 \times 7.4$ & None & $\begin{array}{l}0.5 \mathrm{~cm} \\
\text { bolus }\end{array}$ \\
\hline 2 & No & $\begin{array}{l}\text { Reduced } \\
\text { field size to } \\
4 \mathrm{~cm} \times 6 \mathrm{~cm} \\
\text { for doses } \\
\# 6-7\end{array}$ & Co-60 & Co-60 & SSD & Single field & N/A & $\begin{array}{l}7 \times 10 \text { for } 5 \\
\text { fractions, } \\
4 \times 6 \text { for } 2 \\
\text { fractions }\end{array}$ & $\begin{array}{l}\text { Lead block in } \\
\text { front of } \\
\text { tongue. Gel } \\
\text { agent inside } \\
\text { cheek }\end{array}$ & None \\
\hline 3 & No & No & $4 \mathrm{MV}$ & Clinac 4 & SAD & $\begin{array}{l}\text { Parallel } \\
\text { opposed }\end{array}$ & Equal & $5 \times 8$ & None & None \\
\hline 4 & No & No & Co-60 & Co-60 & SSD & Single field & N/A & $6 \times 8$ & $\begin{array}{l}\text { Pink bolus in } \\
\text { mouth and } \\
\text { superflab } \\
\text { between } \\
\text { gingiva and } \\
\text { lips }\end{array}$ & None \\
\hline 5 & No & No & $6 \mathrm{MV}$ & Clinac 2100 & SAD & $\begin{array}{l}\text { Parallel } \\
\text { opposed }\end{array}$ & Equal & $7.4 \times 4.4$ & $\begin{array}{l}\text { Mouth block; } \\
\text { MLC on } 270^{\circ} \\
\text { beam }\end{array}$ & None \\
\hline 6 & $\begin{array}{l}\text { 13-day break } \\
\text { between } \\
\text { fraction \#7 and } \\
\text { \#8 }\end{array}$ & $\begin{array}{l}\text { Fraction \#8 } \\
\text { and \#9 given } \\
\text { at dose of } \\
6 \text { Gy after the } \\
\text { 13-day break }\end{array}$ & $4 \mathrm{MV}$ & Clinac 4 & SSD & Single field & N/A & $5.5 \times 9.5$ & $\begin{array}{l}\text { Wet gauze in } \\
\text { mouth }\end{array}$ & None \\
\hline 7 & Not described & $\begin{array}{l}\text { Not } \\
\text { described }\end{array}$ & $4 \mathrm{MV}$ & Clinac 4 & SAD & $\begin{array}{l}\text { Parallel } \\
\text { opposed }\end{array}$ & Equal & $3.6 \times 11.5$ & $\begin{array}{l}\text { Wedges }\left(15^{\circ}\right) \\
\text { on both } 0 \text { and } \\
180^{\circ} \text { beams }\end{array}$ & $\begin{array}{l}\text { Not } \\
\text { described }\end{array}$ \\
\hline 8 & Not described & $\begin{array}{l}\text { Not } \\
\text { described }\end{array}$ & $4 \mathrm{MV}$ & Clinac 4 & SAD & $\begin{array}{l}\text { Parallel } \\
\text { opposed }\end{array}$ & Equal & $24 \times 12$ & None & None \\
\hline 9 & No & No & $6 \mathrm{MV}$ & Clinac 2100 & SAD & $\begin{array}{l}\text { Parallel } \\
\text { opposed }\end{array}$ & Equal & $8 \times 16$ & None & $1 \mathrm{~cm}$ bolus \\
\hline 10 & $\begin{array}{l}\text { Only received } \\
5 / 6 \text { prescribed } \\
\text { palliative } \\
\text { doses }\end{array}$ & $\begin{array}{l}\text { Record not } \\
\text { available }\end{array}$ & Co-60 & Co-60 & SSD & $\begin{array}{l}\text { Record not } \\
\text { available }\end{array}$ & $\begin{array}{l}\text { Record not } \\
\text { available }\end{array}$ & $\begin{array}{l}\text { Record not } \\
\text { available }\end{array}$ & $\begin{array}{l}\text { Record not } \\
\text { available }\end{array}$ & $\begin{array}{l}\text { Record not } \\
\text { available }\end{array}$ \\
\hline 11 & $\begin{array}{l}\text { Last treatment } \\
\text { not given }\end{array}$ & $\begin{array}{l}\text { Changed } \\
\text { plan after 1st } \\
\text { fraction }\end{array}$ & $6 \mathrm{MV}$ & Clinac 2100 & SAD & $\begin{array}{l}\text { Single field } \\
\text { for first } \\
\text { fraction, } \\
\text { then } \\
\text { parallel } \\
\text { opposed }\end{array}$ & Equal & $\begin{array}{l}13.4 \times 13.1 \\
\text { then } \\
11.4 \times 8.4\end{array}$ & $\begin{array}{l}\text { First fraction: } \\
\text { MLC, } \\
\text { Subsequent } \\
\text { fractions: MLC } \\
\text { on both fields, } \\
\text { with wedge } \\
\left(45^{\circ}, \text { left) on }\right. \\
210 \text {; mouth } \\
\text { block }\end{array}$ & $\begin{array}{l}1 \mathrm{~cm} \text { on } \\
\text { angle } 210 \\
\text { field }\end{array}$ \\
\hline 12 & No & $\begin{array}{l}\text { First fraction } \\
\text { received } 3 \mathrm{~Gy}\end{array}$ & $4 \mathrm{MV}$ & Clinac 4 & SSD & Single field & N/A & $6.5 \times 4.5$ & None & $\begin{array}{l}0.5 \mathrm{~cm} \\
\text { bolus }\end{array}$ \\
\hline 13 & No & $\begin{array}{l}2 \text { treatments } \\
\text { on same day } \\
\text { (fraction } \\
11-12 \text { ) }\end{array}$ & $6 \mathrm{MV}$ & Clinac 2100 & SAD & $\begin{array}{l}2 \text { field: } 90 \\
\text { and } 275 \\
\text { beam } \\
\text { angles }\end{array}$ & $\begin{array}{l}1.5 \text { (beam } \\
\text { angle 275), } \\
0.5 \text { (beam } \\
\text { angle 90) }\end{array}$ & $\begin{array}{l}9 \times 13.1 \\
\text { and } \\
8.9 \times 13.1\end{array}$ & $\begin{array}{l}\text { Mouth block; } \\
\text { wedge } \\
15^{\circ} \text { (right) on } \\
90^{\circ} \text { beam }\end{array}$ & $\begin{array}{l}\text { Wet gauze } \\
0.5 \mathrm{~cm}\end{array}$ \\
\hline
\end{tabular}

$M V$, megavoltage; SSD, source to surface distance; $S A D$, source to axis distance; $M L C$, multi-leaf collimator. 


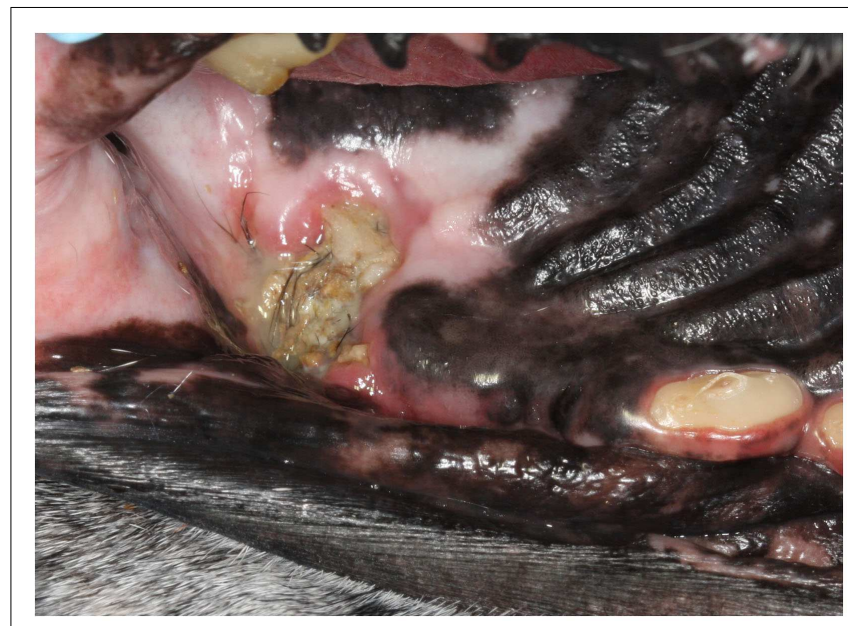

FIGURE 1 | An intraoral photograph of the dog in dorsal recumbency with ORNJ of the left maxilla (Case 5). There is an area of soft tissue necrosis at the level of missing left maxillary first molar tooth with underlying exposed necrotic bone covered partially by debris and hair. Note also the severe abrasion of all remaining teeth.

histopathological evaluation of the right rostral mandible revealed osteonecrosis and SCC, which was removed with tumor-free and osteonecrosis-free margins. Review of the slides confirmed that primary lesion was CAA and the later SCC. Since the time for malignant transformation (5) was short, this may represent de novo tumor development.

In three dogs (including one dog with two ORNJ sites), the lesions healed after surgical intervention (one rim excision, one segmental and later unilateral rostral mandibulectomy, two débridement procedures). Two additional dogs received several débridement procedures to remove all grossly non-vital tissue to achieve stable or slowly progressive disease, or débridement was combined with hyperthermia. One patient was managed medically for 6 months prior to euthanasia. In one dog, progressive disease required another débridement procedure, but the dog was euthanized due to perioperative complications, and in another two dogs euthanasia was suggested due to the extent of the disease and poor quality of life. Four dogs were lost to follow-up.

\section{DISCUSSION}

The present study describes RT and associated treatments employed in a series of 13 dogs treated for odontogenic or nonodontogenic oral tumors. It further focuses on the characteristics of the ONJ/ORNJ lesions that later developed, especially the site of ONJ/ORNJ in relation to the primary tumor, time to onset, and duration of the necrotic lesion, imaging, and histopathology findings.

Radiotherapy alone can provide good tumor control in dogs depending on tumor size and type (2-4), and local control and survival time may be improved by combining RT with radiation sensitizers, surgery, or chemotherapy (2). Some studies suggest that chemotherapy use coincident with radiation may increase the risk of ORNJ development $(1,7)$. Conventional definitive RT in veterinary patients normally employs radiation fractions of

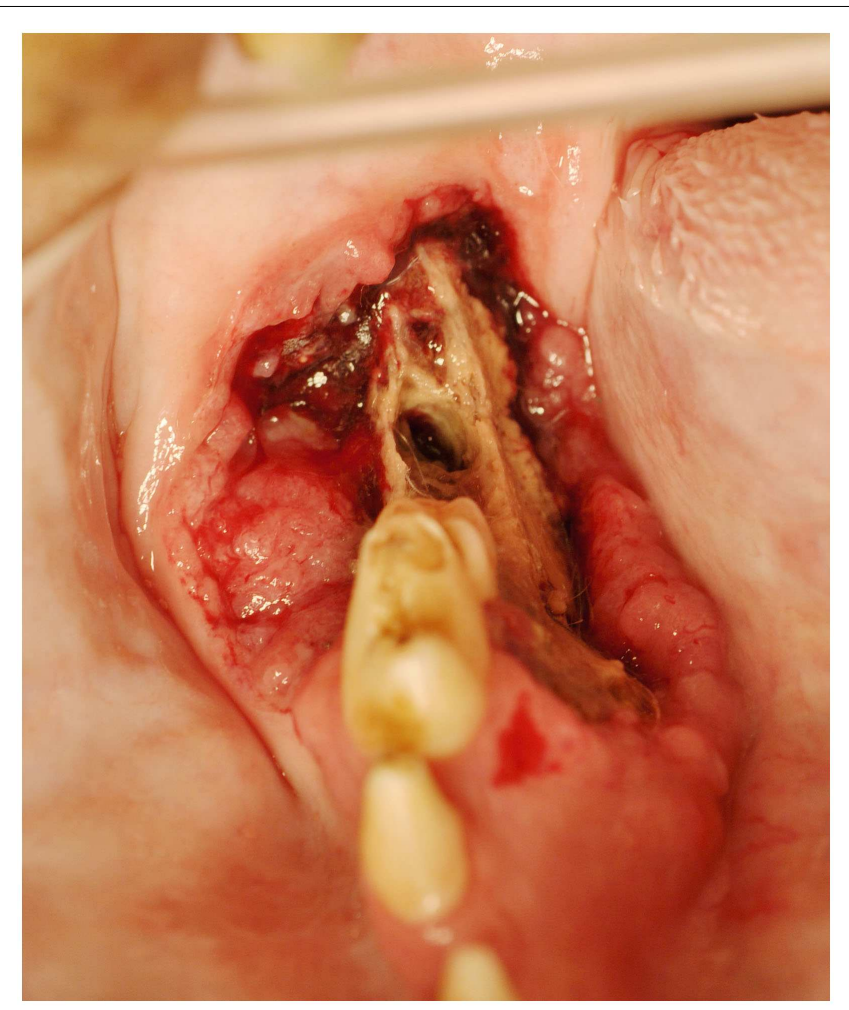

FIGURE 2 |An intraoral photograph of the dog in sternal recumbency with ONJ of the right mandible (Case 9). There is an extensive area of soft tissue necrosis with underlying exposed necrotic bone noted from the distal root of the right mandibular first molar tooth to the level of missing right mandibular third molar tooth. Note an uncomplicated crown fracture of the right mandibular first molar tooth. There is a soft tissue mass noticeable at the buccal aspect of the lesion, suggestive of neoplasia. Biopsy confirmed recurrent SCC.

2-3.2 Gy once daily, 5 days a week, over 16-25 fractions (18). However, palliative RT protocols for veterinary patients have a wider variety of dosing schemes, with fraction sizes of 4-9 Gy given over a week or once weekly for 3-4 weeks being commonly used (19). RT does induce changes in healthy cells and tissues, and both deterministic and stochastic effects can occur (9). ORN is believed to be a deterministic effect, where below a certain threshold dose no effect is observed (9). In human patients, ORNJ is unlikely to occur if the radiation dose delivered by conventional RT is below $60 \mathrm{~Gy}$ $(1,6,20)$. The risk of ORNJ in humans increases with the increased radiation dose $(1,6,20)$. It is notable that the protocols used for RT in humans result in a much higher total dose to the tissue compared to veterinary protocols that normally do not exceed $60 \mathrm{~Gy}$. Despite the lower total doses used in veterinary patients, ORN is seen in patients receiving doses of 32-48 Gy. The evident ORN at lower doses may be due to the higher dose per fraction (e.g., human patients often receive doses $<2 \mathrm{~Gy}$ (7) for definitive protocols compared to veterinary patients that often receive 3-4 Gy for definitive therapy, resulting in a similarly high biologically effective dose despite the lower total dose delivered to dogs). Veterinary patients who have gross disease at the time of RT may also have tumor regression or tumor necrosis resulting in bone exposure, whereas 


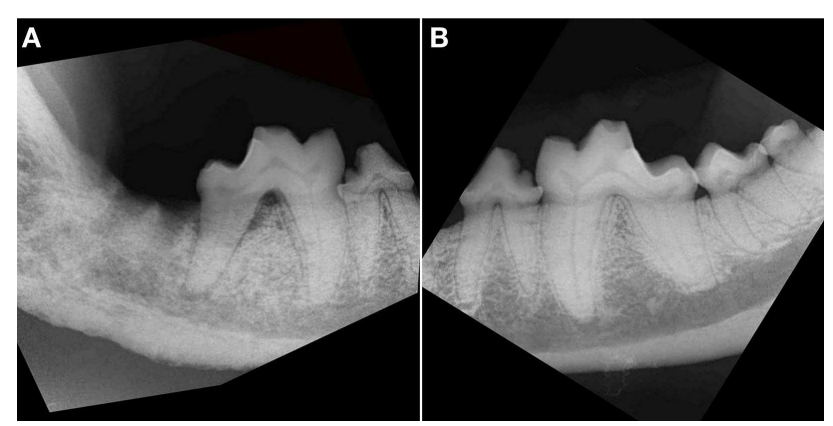

FIGURE 3 | Intraoral radiograph, lateral view of the right (A) and left (B) caudal mandible of the dog in Figure 2. (A) There is an extensive area (all bone visible on the radiograph) of bony destruction, dominated by combined moth-eaten and permeative patterns of bone loss on the right mandible. Note also the generalized loss of definition of the mandibular canal, with a solid periosteal reaction, most prominent at the level of the distal root of right mandibular first molar tooth and missing right second and third molar teeth. There is horizontal and vertical bone loss at the distal root of the fractured right mandibular first molar tooth with furcation involvement. (B) Radiograph of the healthy left mandible shows minor horizontal bone loss at the mesial aspect of the left mandibular fourth premolar tooth and second molar tooth. There is a crown fracture of the left mandibular first molar tooth.

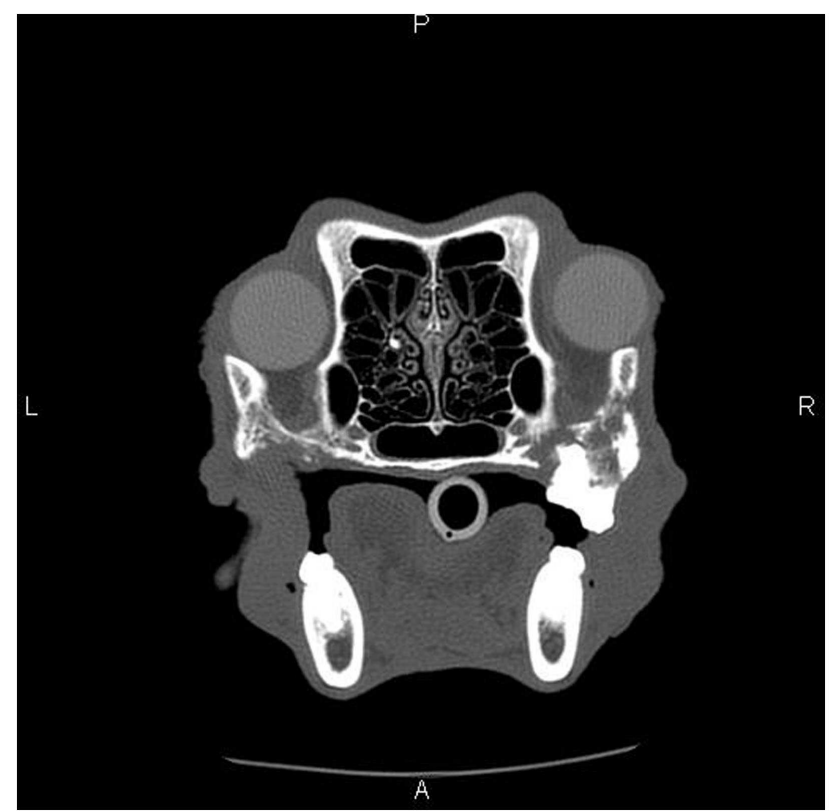

FIGURE 4 | Skull CT of the dog (Case 5) showing the section at the level of maxillary molar teeth. There is extensive irregular osteolysis of the right caudal maxilla, palatine bone, and the rostral aspect of the zygomatic arch. There is remodeling of the caudal maxilla, palatine bone, and rostral zygomatic arch on the left side, where previous osteonecrosis was described (Figure 1). There is a missing left maxillary first molar tooth

patients with a healthy mucosal lining after surgery tend to have less bone exposure. This bone exposure could also contribute to bone lysis, and infection. In human studies, Co-60 RT (6), high

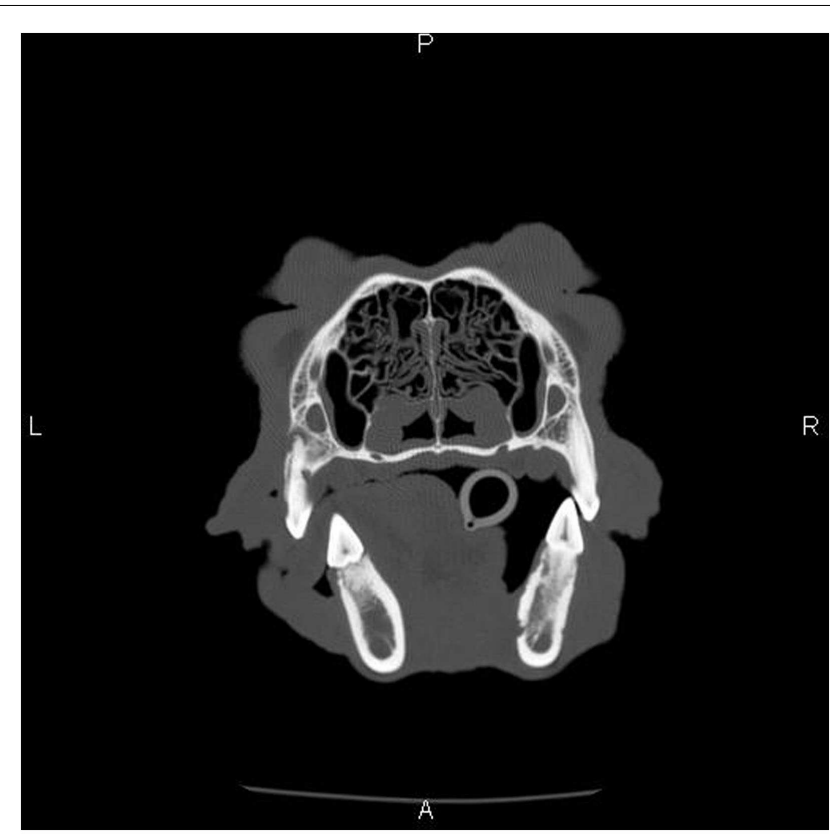

FIGURE 5 | Skull CT of the dog (Case 3) showing the section at the level of mandibular first molar teeth. There is a diffuse thinning and multifocal pitting osteolysis of the cortical bone of the right mandibular body, and the changes are most pronounced in the medial cortex.

total doses, short regimens using higher doses per fraction, and large field sizes are all associated with an increased risk of ORNJ $(1,20)$. Notably, the use of megavoltage RT results in reduced frequency of ORNJ compared to lower-energy, orthovoltage therapy (9). There is also evidence that continuous hyperfractionated accelerated RT possesses less risk for ORNJ development, although these protocols are not easily adapted to veterinary patients due to anesthesia and time constraints, and deliver smaller fractions to a low total dose $(1,7,20)$. Definitive RT (without surgery) and adjunctive RT pose similar risks of developing ORN in humans (7). In this study, corticosteroid use during RT was employed to reduce the RT-induced inflammation of the oral mucosa and skin in seven of the dogs in this study. In a review looking at possible risks and predisposing factors, corticosteroids were found to reduce the risk of ORNJ by $96 \%$ in humans (1).

Upon presentation, the dogs in the present study had lesions in the oral cavity that were clinically described as soft tissue necrosis of variable extent with underlying exposed necrotic bone, warranting a clinical diagnosis of ONJ or, specifically, ORNJ if no tumor was noted (1). Although dogs $<7$ years of age were found to have a significantly higher risk of ORN development at appendicular sites in one study (9), all dogs in the present study were $>8$ years old. RT may have been selected rather than surgery in these older dogs as a less invasive treatment option, particularly for advanced, caudally located tumors. In such cases, curative-intent surgical resection is difficult or impossible; however, prolonged progression free intervals following RT are reported in dogs with large odontogenic tumors (3). The size of the tumors and degree of bone invasion may also influence ORNJ development in humans (1), although 


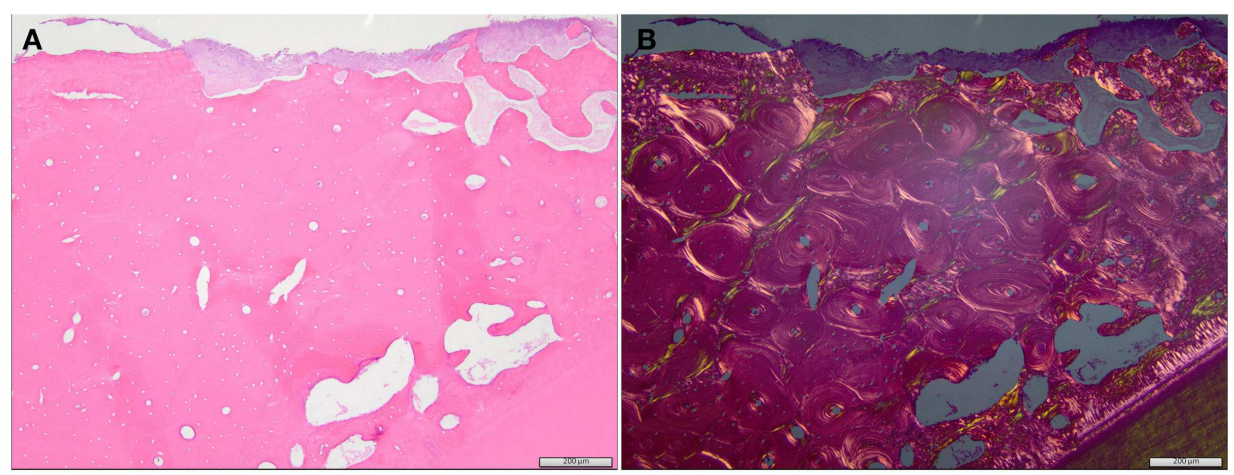

FIGURE 6 | Regular (A) and polarized (B) histological image of the specimen from the dog with an ORNJ lesion of the mandible (Case 3). (A) The alveolar bone comprises primarily lamellar, osteonal bone with limited interstitial woven bone. The bone is necrotic, as evidenced by diffuse, empty osteocyte lacunae, and attached basophilic to eosinophilic biofilm (top of image). Multifocal, irregularly spaced, and shaped our case series included both small (T1) and large (T2, T3) tumors with and without bone involvement.

All but one of the ONJ/ORNJ lesions were found on the site of tumor irradiation or in the jaw closest to the irradiated tumor. Most human patients also develop ORNJ ipsilateral to the tumor inside the radiation field. However, bilateral involvement or involvement of the contralateral jaw may occur $(21,22)$, as also noted in our cases. It is likely that the exit radiation dose contributes to ORNJ in cases that develop bilateral or contralateral ORNJ after receiving a single field of radiation. In cases receiving the common "parallel opposed" radiation technique, wherein radiation beams are delivered from each side of the body, it is likely that both the entrance and exit dose of the beams contribute to development of ipsilateral, contralateral, or bilateral ORNJ lesions. In humans, higher susceptibility of the mandible, and especially the molar region, for ORNJ development is reported $(1,6,7)$. Our study had a similar pattern of caudal location, but site predilection cannot be concluded from the present study.

The time to ORNJ onset varied greatly among dogs (3-44 months after start of RT), which is similar (1-69 months) to observations for mandibular ORN in humans (6). In human, ORNJ typically develops during the first 4-36 months after RT; the risk of developing ORNJ, however, remains for life (1). Because dogs have a life span considerably shorter than that of humans, it is difficult to project how long the risk of developing ORNJ remains in the veterinary population.

None of the dogs in this study had undergone major surgery before RT. Importantly, surgery performed immediately before RT may increase the risk of ORN development in humans (7). In at least three cases, however, ORNJ started after dental extractions. Post-RT surgery, and especially dental extractions in the radiation field, is considered one of the most important predisposing factors for ORNJ development in humans $(1,6,14,23)$, although an incidence of only $2 \%$ per tooth extracted has been reported (14). Reports in veterinary medicine are scarce, but dogs with intranasal neoplasia that had surgery performed after accelerated resorption bays are present within the alveolar bone (clear spaces/osteoporosis). Tooth dentin and a thin rim of cementum are evident at the bottom of the image. These eosinophilic matrices are more evident in the polarized image (B). A periodontal ligament is absent between the cementum and adjacent alveolar bone, consistent with ankylosis of the tooth.

RT were more likely to develop ORN (24). It has been suggested for human patients that comprehensive oral/dental examination and dental extractions of all unrestorable or periodontally affected teeth should be performed at least $7-10$ days before RT, and ideally 3 weeks before RT to allow healing $(1,6-8,23)$. However, protocols for pre-RT dental evaluation have not been well established (7), even though dental status of patients scheduled for RT treatment may be poor (8).

Periodontal disease is a very common dental disease in middleaged to older dogs (25); however, routine oral and dental evaluation and dental treatment were not commonly performed in the dogs included in this study. Ideally, oral/dental evaluation and treatment should be performed prior to initiating RT. If dental treatment is required during or after RT, surgical trauma should be minimized and dogs receiving radiation to the oral cavity, nasal cavity, or neighboring tissues should be managed by experienced oral surgeons (23). Preoperative and postoperative use of topical antiseptics and systemic antibiotics have also been widely employed, but direct evidence that such medical treatment reduces the incidence of ORNJ is currently lacking $(14,23)$. Human studies suggest that oral hygiene should be maximized during RT with the use of topical antiseptic mouthwashes (23); these washes were rarely performed in the dogs reported in this study.

The duration of bone exposure (1) may be very difficult to determine in dogs because it may go unnoticed by the owner for extended periods of time. This issue has also been debated in the human literature, and some authors suggest that bone exposure should be present for at least 1-3 months to prevent over diagnosis of ORNJ $(1,12)$. With only a brief period of observation, a clinician may erroneously diagnose ORNJ in a patient with mucositis or mucosal radionecrosis with exposed but not necrotic underlying bone (1). However, human ORNJ diagnostics suggest that if radiographic changes in the bone are observed at the first visit, a diagnosis of ORNJ can be made regardless of the period of bone exposure (12). 
Imaging, including dental radiography and $\mathrm{CT}$, can be very useful to further investigate ORNJ lesions (1). Dental radiographs of the lesions in advanced stages, when over $30 \%$ of bone mineral content is lost, usually reveal a mixed radiopaque/radiolucent lesion, representing bone destruction (1) similar to the present study. However, plain radiographs usually underestimate the extent of damaged bone and do not correlate with the clinical status (1, 12). A clear line of demarcation between devitalized and vital bone may not be observed (1), which is in agreement with the present report. CT has similar limitations (1) with the earliest osseous imaging findings of ORNJ being cortical defects and trabecular disorganization, air pockets, fractures, and sequestra formation in severely advanced lesions $(21,26)$. Even with imaging, the diagnosis of osteonecrosis may still be difficult, and its definition has been debated in human literature (1). During surgery, bleeding bone can provide a helpful guide to defining the extent of the necrosis (1), but histopathology is the ultimate diagnostic tool to confirm osteonecrosis $(1,13,27)$. Histopathological examination reveals changes typical of a hypovascular and hypocellular/fibrotic tissue $(1,13,27)$, which have been observed in the present study. Importantly, histopathology is also needed to rule out infection/osteomyelitis. In humans, microorganisms are considered to play a minor role in the pathophysiology of ORNJ ( 1 , 27), but they may cause a superimposed infection of ORNJ (21, 27). Also, histopathology is necessary to evaluate persistence or recurrence of the neoplasia. In the present study, five dogs demonstrated evidence of neoplasia, which may have contributed to the osteonecrosis $(1,9)$.

Due to the retrospective nature of the study, certain limitations exist. First, histopathological confirmation of osteonecrosis was not available for all dogs. However, of the seven dogs lacking histopathology review of the clinically diagnosed ONJ lesion, and with an additional three dogs lacking bone in the samples, imaging was available for six. In these cases, the imaging confirmed bony involvement in five cases, supporting the clinical diagnosis of osteonecrosis (12). In one dog where no bony involvement was seen radiographically, progressive disease was observed over 1.5 months without evidence of tumor recurrence, which was most consistent with ORNJ. Also, of the 13 dogs included in this study, 6 had demonstrated evidence of tumor persistence or recurrence, which likely contributed to the osteonecrosis.

\section{CLINICAL RELEVANCE}

The clinical consequences of ORNJ are significant, and treatment may not always be possible due to the extent and severity of the lesion. Although ORNJ appears to be a rare complication, clients should be advised of possible development of ORNJ following RT. In cases that are surgically amenable, especially smaller tumors, surgery should be considered a first line treatment, as complete removal of oral tumors can result in cure (28) with minimal effect on function and appearance (29). In addition, with the development of new reconstructive techniques (30), function and cosmesis can often be preserved even with larger resections. Finally, until with further studies we better understand ORNJ development in animals, we suggest an oral exam and treatment to be performed prior to RT for an oral tumor in dogs, as is the standard practice in human medicine.

\section{AUTHOR CONTRIBUTIONS}

All authors substantially contributed to the conception of the work, acquisition, analysis, and interpretation of the data. Agreement was reached by all authors for all aspects of the work in ensuring that questions related to the accuracy and integrity of all parts of the work are appropriately investigated and resolved. Ana Nemec drafted the manuscript, which was critically revised for important intellectual content and the final version approved to be published by all authors.

\section{REFERENCES}

1. Chrcanovic BR, Reher P, Sousa AA, Harris M. Osteoradionecrosis of the jaws - a current overview - part 1: physiopathology and risk and predisposing factors. Oral Maxillofac Surg (2010) 14:3-16. doi:10.1007/s10006-009-0198-9

2. Liptak JM, Withrow SJ. Cancer of the gastrointestinal tract. Section A: oral tumors. In: Withrow SJ, Vail DM, editors. Withrow \& MacEwen's small animal clinical oncology. St. Louis, MO: Saunders Elsevier (2007). p. 455-75.

3. Theon AP, Rodriguez C, Griffey S, Madewell BR. Analysis of prognostic factors and patterns of failure in dogs with periodontal tumors treated with megavoltage irradiation. J Am Vet Med Assoc (1997) 210:785-8.

4. Theon AP, Rodriguez C, Madewell BR. Analysis of prognostic factors and patterns of failure in dogs with malignant oral tumors treated with megavoltage irradiation. J Am Vet Med Assoc (1997) 210:778-84.

5. McEntee MC, Page RL, Theon A, Erb HN, Thrall DE. Malignant tumor formation in dogs previously irradiated for acanthomatous epulis. Vet Radiol Ultrasound (2004) 45:357-61. doi:10.1111/j.1740-8261.2004.04067.x

6. Lee IJ, Koom WS, Lee CG, Kim YB, Yoo SW, Keum KC, et al. Risk factors and dose-effect relationship for mandibular osteoradionecrosis in oral and oropharyngeal cancer patients. Int J Radiat Oncol Biol Phys (2009) 75:1084-91. doi:10.1016/j.ijrobp.2008.12.052

7. Nabil S, Samman N. Risk factors for osteoradionecrosis after head and neck radiation: a systematic review. Oral Surg Oral Med Oral Pathol Oral Radiol (2012) 113:54-69. doi:10.1016/j.tripleo.2011.07.042

8. Niewald M, Fleckenstein J, Mang K, Holtmann H, Spitzer WJ, Rube C. Dental status, dental rehabilitation procedures, demographic and oncological data as potential risk factors for infected osteoradionecrosis of the lower jaw after radiotherapy for oral neoplasms: a retrospective evaluation. Radiat Oncol (2013) 8:227. doi:10.1186/1748-717X-8-227

9. Hosoya K, Poulson JM, Azuma C. Osteoradionecrosis and radiation induced bone tumors following orthovoltage radiation therapy in dogs. Vet Radiol Ultrasound (2008) 49:189-95. doi:10.1111/j.1740-8261.2008.00349.x

10. Thrall DE. Orthovoltage radiotherapy of acanthomatous epulides in 39 dogs. $J$ Am Vet Med Assoc (1984) 184:826-9.

11. D'Souza J, Lowe D, Rogers SN. Changing trends and the role of medical management on the outcome of patients treated for osteoradionecrosis of the mandible: experience from a regional head and neck unit. Br J Oral Maxillofac Surg (2014) 52:356-62. doi:10.1016/j.bjoms.2014.01.003

12. Karagozoglu KH, Dekker HA, Rietveld D, de Bree R, Schulten EA, Kantola S, et al. Proposal for a new staging system for osteoradionecrosis of the mandible. Med Oral Patol Oral Cir Bucal (2014) 19:433-7. doi:10.4317/medoral.19623

13. Lyons A, Ghazali N. Osteoradionecrosis of the jaws: current understanding of its pathophysiology and treatment. Br J Oral Maxillofac Surg (2008) 46:653-60. doi:10.1016/j.bjoms.2008.04.006

14. Nabil S, Samman N. Incidence and prevention of osteoradionecrosis after dental extraction in irradiated patients: a systematic review. Int J Oral Maxillofac Surg (2011) 40:229-43. doi:10.1016/j.ijom.2010.10.005

15. Arzi B, Verstraete FJ. Clinical staging and biopsy of maxillofacial tumors. In: Verstraete FJ, Lommer MJ, editors. Oral and Maxillofacial Surgery in Dogs and Cats. Edinburgh: Saunders Elsevier (2012). p. 373-80.

16. Keyerleber MA, McEntee MC, Farrelly J, Podgorsak M. Completeness of reporting of radiation therapy planning, dose, and delivery in veterinary radiation oncology manuscripts from 2005 to 2010. Vet Radiol Ultrasound (2012) 53:221-30. doi:10.1111/j.1740-8261.2011.01882.x

17. Dobson H, Friedman L. Radiologic interpretation of bone. In: Summer-Smith G, editor. Bone in Clinical Orthopedics. New York, NY: Thieme (2002). p. 175-202.

18. McEntee MC. Veterinary radiation therapy: review and current state of the art. J Am Anim Hosp Assoc (2006) 42:94-109. doi:10.5326/0420094 
19. Tan-Coleman B, Lyons J, Lewis C, Rosenberg M, Ruiz A. Prospective evaluation of a 5 x 4 Gy prescription for palliation of canine nasal tumors. Vet Radiol Ultrasound (2013) 54:89-92. doi:10.1111/j.1740-8261.2012.01984.x

20. Studer G, Gratz KW, Glanzmann C. Osteoradionecrosis of the mandibula in patients treated with different fractionations. Strahlenther Onkol (2004) 180:233-40. doi:10.1007/s00066-004-1171-z

21. Hamilton JD, Lai SY, Ginsberg LE. Superimposed infection in mandibular osteoradionecrosis: diagnosis and outcomes. J Comput Assist Tomogr (2012) 36:725-31. doi:10.1097/RCT.0b013e3182702f09

22. Thorn JJ, Hansen HS, Specht L, Bastholt L. Osteoradionecrosis of the jaws: clinical characteristics and relation to the field of irradiation. J Oral Maxillofac Surg (2000) 58:1088-95. doi:10.1053/joms.2000.9562

23. McLeod NM, Bater MC, Brennan PA. Management of patients at risk of osteoradionecrosis: results of survey of dentists and oral \& maxillofacial surgery units in the United Kingdom, and suggestions for best practice. Br J Oral Maxillofac Surg (2010) 48:301-4. doi:10.1016/j.bjoms.2009.06.128

24. Adams WM, Bjorling DE, McAnulty JE, Green EM, Forrest LJ, Vail DM. Outcome of accelerated radiotherapy alone or accelerated radiotherapy followed by exenteration of the nasal cavity in dogs with intranasal neoplasia: 53 cases (19902002). J Am Vet Med Assoc (2005) 227:936-41. doi:10.2460/javma.2005.227.936

25. Harvey CE, Shofer FS, Laster L. Association of age and body weight with periodontal disease in North American dogs. J Vet Dent (1994) 11:94-105.

26. Saito N, Nadgir RN, Nakahira M, Takahashi M, Uchino A, Kimura F, et al. Posttreatment CT and MR imaging in head and neck cancer: what the radiologist needs to know. Radiographics (2012) 32:1261-84. doi:10.1148/rg.325115160

27. Marx RE, Tursun R. Suppurative osteomyelitis, bisphosphonate induced osteonecrosis, osteoradionecrosis: a blinded histopathologic comparison and its implications for the mechanism of each disease. Int J Oral Maxillofac Surg (2012) 41:283-9. doi:10.1016/j.ijom.2011.12.016
28. Fulton AJ, Nemec A, Murphy BG, Kass PH, Verstraete FJ. Risk factors associated with survival in dogs with nontonsillar oral squamous cell carcinoma 31 cases (1990-2010). J Am Vet Med Assoc (2013) 243:696-702. doi:10.2460/javma.243. 5.696

29. Lommer MJ, Verstraete FJM. Principles of oral oncologic surgery. In: Verstraete FJM, Lommer MJ, editors. Oral and Maxillofacial Surgery in Dogs and Cats. Edinburgh: Saunders Elsevier (2012). p. 423-30.

30. Arzi B, Verstraete FJ, Huey DJ, Cissell DD, Athanasiou KA. Regenerating mandibular bone using rhBMP-2: part 1-immediate reconstruction of segmental mandibulectomies. Vet Surg (2014). doi:10.1111/j.1532-950X.2014.12123.x

Conflict of Interest Statement: The authors declare that the research was conducted in the absence of any commercial or financial relationships that could be construed as a potential conflict of interest.

Received: 05 February 2015; accepted: 19 March 2015; published online: 01 April 2015. Citation: Nemec A, Arzi B, Hansen K, Murphy BG, Lommer MJ, Peralta S and Verstraete FJM (2015) Osteonecrosis of the jaws in dogs in previously irradiated fields: 13 cases (1989-2014). Front. Vet. Sci. 2:5. doi: 10.3389/fvets.2015.00005

This article was submitted to Veterinary Dentistry and Oromaxillofacial Surgery, a section of the journal Frontiers in Veterinary Science.

Copyright (c) 2015 Nemec, Arzi, Hansen, Murphy, Lommer, Peralta and Verstraete. This is an open-access article distributed under the terms of the Creative Commons Attribution License (CC BY). The use, distribution or reproduction in other forums is permitted, provided the original author(s) or licensor are credited and that the original publication in this journal is cited, in accordance with accepted academic practice. No use, distribution or reproduction is permitted which does not comply with these terms. 